A Scoping Review of Research on Food Design Interventions to Improve Nutrition in Adults:

A Focus on Food Shaping with Implications for Older Adults with Swallowing Disorders

Lily Chen, MS, RDN, APD, FAND (PhD Candidate)

University of Technology Sydney 


\section{A Scoping Review of Research on Food Design Interventions to Improve Nutrition in Adults: A Focus on Food Shaping with Implications for Older Adults with Swallowing Disorders}

\section{The Importance of Access to Good Nutrition in Older Adults}

The role of food and nutrition in ageing is vital to both maintaining health and quality of life. Nutrition is essential to provide essential building blocks for health throughout the lifespan. Furthermore, nutritious food is not only critical to one's physiological well-being, but also contributes to social, cultural, and psychological quality of life (Bernstein \& Munoz, 2012). Improving access to good nutrition decreases risk of chronic disease, slows disease progression and reduces related symptoms (Bernstein \& Munoz, 2012).

Ageing may impede the older adult's ability to obtain good nutrition. Moreover, agerelated factors such as sense of smell and taste, ability to chew and swallow, gastrointestinal and bowel function can impact an older person's quality of life (Amarantos, Martinez, \& Dwyer, 2001). Changes in smell and taste are caused by several functional changes and medical states associated with aging, including: changes to taste bud number and structure, dry mouth and throat, disease states, medications, and surgical interventions (Nieuwenhuizen, Weenen, Rigby, \& Hetherington, 2010). The senses of taste and smell are essential for the initial phase of digestion that triggers the release of salivary, gastric, pancreatic, and intestinal secretions which all contribute to digestion (Nieuwenhuizen et al., 2010). Thus, the loss of smell and taste is concerning as it impacts enjoyment and appetite, along with access to nutrition.

\section{Swallowing difficulty in older people}

Dysphagia (swallowing difficulty) can significantly impact on a person's nutrition status. Long term care residents, in particular, are at a high risk of experiencing some level of swallowing difficulty (Namasivayam-MacDonald \& Riquelme, 2019). Chewing difficulty reflects difficulty in the oral phase of the swallow and may also present as a barrier for the older adult. The particle size of a swallowed bolus is larger in individuals with decreased dentition or wearing dentures due to lower masticatory efficiency (Matsuo \& Fujishima, 2020). This may place individuals with poor dentition at a higher risk of choking.

The role of the speech pathologist is essential for management and treatment of dysphagia. Older people might or might not adapt to swallowing difficulties by taking smaller bites, eating more slowly, or avoiding food and liquids that are difficult to consume without the knowledge that a swallowing difficulty exists (Namasivayam-MacDonald \& Riquelme, 2019). Consequently, by the time the individual seeks or is referred for a speech pathology assessment of swallowing, the level of swallowing difficulty may have become unmanageable and intertwined with other co-morbidities such as weight loss or malnutrition (NamasivayamMacDonald \& Riquelme, 2019).

Individuals with dysphagia or swallowing difficulties may require specific texturemodified food. Family members, support workers, and staff in residential care settings may 
struggle with the provision of texture-modified food due to a lack of standardisation in diet terminology resulting in different interpretations of what is meant by a 'soft', 'cut up', 'minced' or 'pureed' diet (Hemsley, Palmer, Kouzani, Adams, \& Balandin, 2019). Textured-modified diets also pose risks to nutrition, in that they may be lower in nutrients than a regular diet which can lead to malnutrition or sarcopenia (Ueshima et al., 2021).

The provision of textured food for older people, residents in aged care settings in particular, can be problematic, with anecdotal reports suggesting that pureed food diets are unappealing and unappetising (Hemsley et al., 2019). Creating an appealing look and texture of foods is important to prevent food rejection and malnourishment (T. Pereira, Barroso, \& Gil, 2021). Food perception is complex and related not only to the look of the food, but also its smell and taste. For example, bitter taste perception is linked to the foundation of human evolution, inherently avoiding harmful substances (L. J. Pereira \& van der Bilt, 2016). Overall, taste perception emerges as the primary factor in consumer perception but is not well understood in the provision of meals to older people in aged care settings (L. J. Pereira \& van der Bilt, 2016).

The dietitian plays a critical role in providing nutrition care and interventions for the older adult. Nutrition plays a pivotal role in the aged care setting to promote enhanced health outcomes. A dietitian's services include assessing nutrition status, developing appropriate nutrition interventions, monitoring and evaluating outcomes (Dorner \& Friedrich, 2018). The dietitian also supports the individual adult with a range of interventions including nutrition education and specialised food production to provide personalised care.

With both speech language pathology and dietetics being essential health disciplines involved in the assessment and management of older people in aged care settings, it is important to identify ways to both maximise the safe texture of food while maximising the nutritional aspects of the meal. To date, most research on dysphagia in older people in aged care has focused on their medical and respiratory health, with less of a focus on the impact of modifying food textures on their access to nutrition. Indeed, the recommendation for thickened fluids carries with it a risk of dehydration; and pureed foods risk reducing the nutritional content of the meal owing to dilution or reduction in the types of foods included in the diet, or reduced appetite and lowered intake overall. Both speech pathologists and dietitians have an important inter-disciplinary role in making the person's texture-modified meals appetising and nutritionally beneficial, while reducing the risk of harms of recommending a texture-modified diet.

\section{Food shaping techniques as strategies to improve nutrition in older people}

Health professionals and aged care service providers may strive to reduce the risks to nutrition posed by a texture-modified diet by attending more broadly to elements of food design, specifically food structure shape and presentation on the plate. Recognition of this is seen in the development of food mould technologies (e.g., using metal, glass, or silicon moulds) 
and 3D food printing technologies (e.g., using food as a printable 'ink' in a 3D printer designed to print pureed food shapes).

Pureed consistencies are generally perceived as unappealing due to the resemblance to food prepared for infants (Stahlman et al., 2001). The previously held connotation of the food being suitable for children can lead to disappointment for the older adult, who may also miss

their usual foods. Food moulds are a tool to present pureed food as regular consistency food in a visually appealing way. To date, there is little research examining the feasibility or impact of using spoon shapes, ice-cream scoops, food moulds, piping bags, 3D food printing, or other methods to improve the shape of texture-modified foods as a way to increase access to improved nutrition for older adults with dysphagia. No prior systematic reviews have examined food shaping techniques as a nutritional intervention for older adults, a population at significantly higher risk of having multiple health conditions and for whom quality of life related to mealtimes is increasingly important. This review is needed to help inform strategies to improve nutrition through food shaping. The review findings could inform improvements to food design in aged care settings, and identify gaps in knowledge that could inform future research.

\section{Review Questions}

The aim of this scoping review is to appraise and synthesise the scientific evidence on the use of food shaping interventions aimed at enhancing nutrition in adults. Specifically, the review aims to answer the following questions:

1. What food shaping interventions have been examined as a means to increase access to nutrition for adults, across populations and settings?

2. What food shaping interventions have been examined as a means to increase access to nutrition for older adults (a) with swallowing difficulty or with conditions with high prevalence of swallowing difficulty, or (b) in aged care settings?

\section{Search Terms and Search Strategy}

A key word search will be conducted using the following electronic bibliographic databases: MEDLINE (PubMed), CINAHL, Scopus, Google Scholar, Cochrane and Web of Science.

Various combinations and permutations of a range of key search terms related to food shaping (e.g., spoon shapes, ice-cream scoops, food moulds, piping bags, 3D food printing), nutrition and nutrition interventions, older adults, and dysphagia or swallowing difficulty will be used in the search strategy. The full search strategy is available from authors.

Forward and backward citations will be checked for each included individual study or systematic review to identify any additional studies meeting the inclusion criteria. The publication histories of key authors identified in the search will also be searched by hand. 


\section{Inclusion and exclusion criteria}

A restriction of 20 years will be applied to length of search secondary to the estimated length of time food shaping has been researched.

The search will be restricted to peer reviewed original research and systematic reviews published in English. Any studies located in the search of databases that are also found within included systematic reviews will be retained as individual studies for the review.

This review will include systematic reviews published in peer-reviewed journals in English which report on the intersection of food shaping interventions, nutrition, and adults, including older people living in aged care settings. It will also include empirical research of all types (e.g., observational studies, case studies, case series, experimental and quasi-experimental research, qualitative studies, quantitative studies, and mixed-methods designs).

Any papers that are (a) not in English, are non-peer-reviewed papers, are not original research or systematic review (e.g., narrative review), are full text commentary papers, editorials, or conference abstracts, or (b) not relating to food shaping interventions, nutrition and adults, will be excluded from the review.

\section{Condition or domain being studied}

The domain being studied is the nutrition status of adults and the use of spoon shapes, icecream scoops, food moulds, piping bags, 3D food printing or other methods to improve mealtime safety and enjoyment, along with quality of life, health and wellbeing. Nutrition, or clinical nutrition, is the prevention, diagnosis and management of nutritional and metabolic changes related to acute and chronic diseases and condition caused by a lack of excess of energy and nutrients (macro and micro) as defined by the Academy of Nutrition and Dietetics ("Definition of Terms List," 2021).

\section{Participants/population}

Adults, older people, people living in aged care settings.

\section{Intervention(s), exposure(s)}

The review will focus on the impact and use of nutritional interventions involving any food shaping or enhancements techniques, including spoon shapes, ice-cream scoops, food moulds, piping bags or 3D food printing to enhance the nutrition status.

\section{Main outcome(s)}

This review will investigate outcomes of using food shaping techniques including spoon shapes, ice-cream scoops, food moulds, piping bags, 3D food printing, and any other techniques used to improve the safety or enjoyment of meals for adults.

\section{Study selection}


Identified titles will be screened by one researcher (the first author). After duplicate studies not in English are removed, titles and abstracts will be screened independently by two raters (the first and last author). Any differences of opinion will be resolved by a third rater (the second author). Full texts will be retrieved and rated for inclusion or exclusion in the same manner. A flow diagram using Preferred Reporting Items for Systematic Reviews and Meta-Analyses for Protocols (PRISMA-P) guidelines (Moher et al., 2015) for reporting systematic reviews will be used to report selection process and results.

\section{Data extraction}

The first author will extract data from each study and this will be checked for accuracy by a second researcher. Information extracted from each study will include: author, year, aim, study design, methods, instruments, participants, any participants excluded, major results, outcomes, and directions for future research.

\section{Data analysis}

Our knowledge of the literature and preliminary searches have indicated that a meta-analysis across studies will most likely not be possible in this review, owing to high heterogeneity of the population and lack of controlled trials in this area. For any quantitative data included in studies, descriptive analysis (e.g., frequency, percentages, raw data) will be undertaken. For any qualitative data in the studies, a content thematic approach will be used in order to undertake data synthesis. Synthesis will be structured around the types of intervention described in included studies including the focus and content of interventions. If both qualitative and quantitative data are present, an integrative review will be undertaken utilising both descriptive and qualitative methods. Major and minor themes, and categories of meaning, within and between studies will be explored and presented.

\section{Risk of bias (quality) assessment}

The full texts will be assessed by two authors using the Quality Assessment Tool for Studies with Diverse Designs (QATSDD) (Sirriyeh, Lawton, Gardner, \& Armitage, 2012) to determine quality and relevance for this review. 


\section{References}

Amarantos, E., Martinez, A., \& Dwyer, J. (2001). Nutrition and quality of life in older adults. J Gerontol A Biol Sci Med Sci, 56 Spec No 2, 54-64. doi:10.1093/gerona/56.suppl_2.54

Bernstein, M., \& Munoz, N. (2012). Position of the Academy of Nutrition and Dietetics: food and nutrition for older adults: promoting health and wellness. J Acad Nutr Diet, 112(8), 1255-1277. doi:10.1016/j.jand.2012.06.015

Definition of Terms List. (2021). In: Academy of Nutrition and Dietetics.

Dorner, B., \& Friedrich, E. K. (2018). Position of the Academy of Nutrition and Dietetics: Individualized Nutrition Approaches for Older Adults: Long-Term Care, Post-Acute Care, and Other Settings. J Acad Nutr Diet, 118(4), 724-735. doi:10.1016/j.jand.2018.01.022

Hemsley, B., Palmer, S., Kouzani, A., Adams, S., \& Balandin, S. (2019). Review informing the design of 3D food printing for people with swallowing disorders: Constructive, conceptual, and empirical problems. Paper presented at the HICSS 52: Proceedings of the 52nd Annual Hawaii International Conference on System Sciences.

Matsuo, K., \& Fujishima, I. (2020). Textural Changes by Mastication and Proper Food Texture for Patients with Oropharyngeal Dysphagia. Nutrients, 12(6). doi:10.3390/nu12061613

Moher, D., Shamseer, L., Clarke, M., Ghersi, D., Liberati, A., Petticrew, M., . . Group, P.-P. (2015). Preferred reporting items for systematic review and meta-analysis protocols (PRISMA-P) 2015 statement. Systematic Reviews, 4(1), 1. doi:10.1186/2046-4053-4-1

Namasivayam-MacDonald, A. M., \& Riquelme, L. F. (2019). Presbyphagia to Dysphagia: Multiple Perspectives and Strategies for Quality Care of Older Adults. Semin Speech Lang, 40(3), 227-242. doi:10.1055/s-0039-1688837

Nieuwenhuizen, W. F., Weenen, H., Rigby, P., \& Hetherington, M. M. (2010). Older adults and patients in need of nutritional support: review of current treatment options and factors influencing nutritional intake. Clin Nutr, 29(2), 160-169. doi:10.1016/j.clnu.2009.09.003

Pereira, L. J., \& van der Bilt, A. (2016). The influence of oral processing, food perception and social aspects on food consumption: a review. J Oral Rehabil, 43(8), 630-648. doi:10.1111/joor.12395

Pereira, T., Barroso, S., \& Gil, M. M. (2021). Food Texture Design by 3D Printing: A Review. Foods (Basel, Switzerland), 10(2), 320. doi:10.3390/foods10020320

Sirriyeh, R., Lawton, R., Gardner, P., \& Armitage, G. (2012). Reviewing studies with diverse designs: the development and evaluation of a new tool. J Eval Clin Pract, 18(4), 746-752. doi:10.1111/j.1365-2753.2011.01662.x

Stahlman, L. B., Garcia, J. M., Chambers, E. t., Smit, A. B., Hoag, L., \& Chambers, D. H. (2001). Perceptual ratings for pureed and molded peaches for individuals with and without impaired swallowing. Dysphagia, 16(4), 254-262. doi:10.1007/s00455-001-0084-6

Ueshima, J., Momosaki, R., Shimizu, A., Motokawa, K., Sonoi, M., Shirai, Y., . . Sakai, K. (2021). Nutritional Assessment in Adult Patients with Dysphagia: A Scoping Review. Nutrients, 13(3). doi:10.3390/nu13030778 